\title{
VARIATION OF COMORBIDITIES IN ROMANIA AT THE LAU2 LEVEL, A CROSS-SECTIONAL ANALYSIS \\ Adrian Pana ${ }^{1}$, Bogdan-Vasile Ileanu $^{2}$
}

\begin{abstract}
:
Introduction: Romania is still lagging behind in regard to main health indicators when compared to average values of European Union countries with a disproportionate share of disability due mainly to chronic diseases. There are tools available to quantify the negative health outcomes due to comorbidities, and among them the Elixhauser Index was found to be the best to predict health care utilization and expenditures.

Objective: To measure the extent of comorbidities at the LAU2 level using the Elixhauser Index.

Methods: All relevant comorbidities at the local level were summed-up using the Elixhauser index. This was followed with standardization by population number and then by 10-year age group. Due to large oscillation from one year to another during the 2014-2016 period, the geometrical mean was computed, then few measures of the variation such as decile ratio and variation coefficient were also computed.

Results: High variability of comorbidities is observed at the local level (LAU2). Clusters of comorbidities concentration are found near medical university centers and localities in non-MUC districts located at the furthest distance from the county residential center.

Conclusions: Several factors such as proximity to referral hospitals, increased accessibility to these hospitals due to low control at primary healthcare levels, availability of diagnostic technologies and different diagnostic coding patterns, as well as isolation and unavailability of primary health care services are listed as possible factors that can explain these results.
\end{abstract}

UDC Classification: 614.2, DOI: 10.12955/cbup.v7.1456

Keywords: Comorbidities, Elixhauser Index, Cross-sectional variation, LAU2 unit, Romania

\section{Introduction}

Although key health indicators in Romania had a tendency for improvement in the last few years, values regarding life expectancy at birth, and standardized general mortality rates are still far behind when compared to the average values for European Union countries according to European Health Information Gateway database (EHIG, 2019). According to the Institute of Health Metrics and Evaluation estimations, the top causes of deaths and premature deaths for the Romanian population are related to chronic diseases (IHME, 2019). One in four people declared that they suffer of at least one chronic condition according to a perceived health status survey administered in 2014 on the Romanian population (NIS, 2014), and with the population aging, the share of multi-morbidity is even higher (Brenan et al., 2017).

According to the Global Burden of Diseases studies, the age-specific ratio of observed-to-expected mortality rates for the Romanian population is mainly positive for the younger population and negative for the adult and elderly population (Murray et al., 2017). Also, even though the life expectancy is increasing both at birth and at 65 years old as compared to 1990, the proportion of health adjusted life years is decreasing for the same period (Kyu et al., 2018).

Analyzing the extent of comorbidities or multimorbidity in a population, offers insights not only about the burden of diseases, but also about the potential negative health outcomes according to AlonsoMoran et al. (2015). There are several co-morbidity and multi-morbidity indexes developed in order to quantify and predict negative health outcomes, such as the Charlson Comorbidity Index (Charlson, 1994), the Elixhauser Index (Elixhauser et al, 1998), and the Chronic Diseases Score (Putnam et al., 2002). Out of these indices, the Elixhauser index was the best one to predict health care utilization and expenditures according to $\mathrm{Ou}$ et al. (2012), in a study regarding the comparative performance of performance indices for diabetic Medicaid patients in the United States.

Taking into account the fact that the Romanian population is ageing and has a disproportionate share of disability mainly due to chronic diseases, we decided to analyze the variation of comorbidity at the local level using the Elixhauser index.

\footnotetext{
${ }^{1}$ Bucharest University of Economic Studies; (2) Center for Health Outcomes and Evaluation, Bucharest, Romania, adrian.pana@me.com

${ }^{2}$ Bucharest University of Economic Studies; (2) Center for Health Outcomes and Evaluation, Bucharest, Romania,ileanub@yahoo.com
} 


\section{Data and methodology}

The data used in this study came from different sources. The Statistical Centre of National Institute of Public Health, Romania, provided the hospitalization data. The population data was downloaded from the public portal TEMPO of The Romanian National Institute of Statistics. The distances between the local administrative unit (here LAU2) and the county residential center were computed by the authors using the free Google distance application programming interface (API) tool. The reference period was 2014-2016.

First, we computed a local LAU2 Elixhauser comorbidity index. Starting from the methodology proposed by Elixhauser for one patient, we defined the $E L X_{i t}$ by counting all the comorbidities for all hospitalized patients from a particular locality $\mathrm{i}$, in a calendar year $\mathrm{t}$

$$
E L X S_{i t}=\sum_{j=1}^{n_{i}} \sum_{k=1}^{30} x_{k j i t}
$$

where $\mathrm{k}=1 . .30$ represent a counter for comorbidities, $\mathrm{j}$ represents a counter for the number of patients and $x_{k j i t}$ represents the number of patients in year $\mathrm{t}$, from locality $\mathrm{i}$ which have a particular comorbidity k. In each LAU2 we consider that there are $n_{i}$ patients with comorbidities. Since the number of comorbidities is directly correlated with the age of the patient and the total score will be also dependent by the number of hospitalized patients, we decided to standardize the score in two stages. First, we divided the ELIXS it $_{\text {b }}$ by total residential population in year $t$ and locality i. Then we computed the age-standardized Elixhauser comorbidity index in order to remove age effects in crosssectional comparison. The standardization was made by 10-year age groups, from 0-9 year group to $80+$, using as standard population the residential population of the LAU2 from 2011, the last year of the National Census. As a result, the ELIXS standardized index has the following relation:

$$
E_{\text {LIX }}^{\text {age-std }}=\sum_{a=1}^{9} w_{a} \frac{X_{\text {ait }}}{P_{\text {ait }}}
$$

where $w_{a}$ is the weight of the population from age-group a in the total standard population, as defined here, $X_{\text {ait }}$ is the total Elixhauser index from age-group a, locality $\mathrm{i}$ and year $\mathrm{t}$ and $P_{\text {ait }}$ is the residential population from age-group a, year $t$ and locality $i$.

Further, in order to reduce the variation from one year to another we computed one synthetical measure for each locality as a geometrical mean,

$$
E L I X S_{i 2014-2016}^{\text {age-std }}=\sqrt[3]{E L I X S_{i 2014}^{\text {age-std }} * E L I X S_{i 2015}^{\text {age-std }} * E L I X S_{i 2016}^{\text {age-std }}}
$$

With the values of ELIXS achieved, several classical measures such as the Pearson correlation and variation coefficient $(\mathrm{CV})$, decile ratio, were computed in order to analyze variability and its causes. For simplicity, in the next sections, the ELIXS age-std ${ }_{i 2014-2016}$ is called the Elixhauser Index (EI). For a detailed analysis we define two classes of counties: (1) MUC - the counties that have major medical university center. Here we included Bucharest, Cluj, Timis, Iasi and Craiova NUTS-3 units and (2) Non-MUC, formed by the other 37 NUTS-3 units.

\section{Results and Discussion}

According to Figure 1 the variability of the age-standardized Elixhauser Index is important. In the Northwest Region and Southwest Region of Romania, many localities have a very high level of EI. Some other focal points formed by LAU2 units with high level of EI are visible around Medical University Centers like Cluj-Napoca, Iasi and Bucharest. In contrast, a yellow area was delimited in the center of Romania (Brasov, Covasna, Harghita county), in the West (Timis-Arad area) and in the Southeast part, the counties neighboring the Black-Sea. Even if the color shows a very high contrast across the country, looking at the decile values we can observe that the ratio decile 9 vs. decile 1 is below 2. When a correlation analysis is made, absolute values of the Pearson correlation are below 0.1 .

But when the results plotted in the Figure 2 are studied, we observe that in the proximity of Medical University Centers we have an excess of comorbidities. This association decreases slowly by distance until the distance reach about $90 \mathrm{~km}$, equivalent to $1.5-2$ hours of normal driving. More types of behavior not necessarily independent can explain this result: 


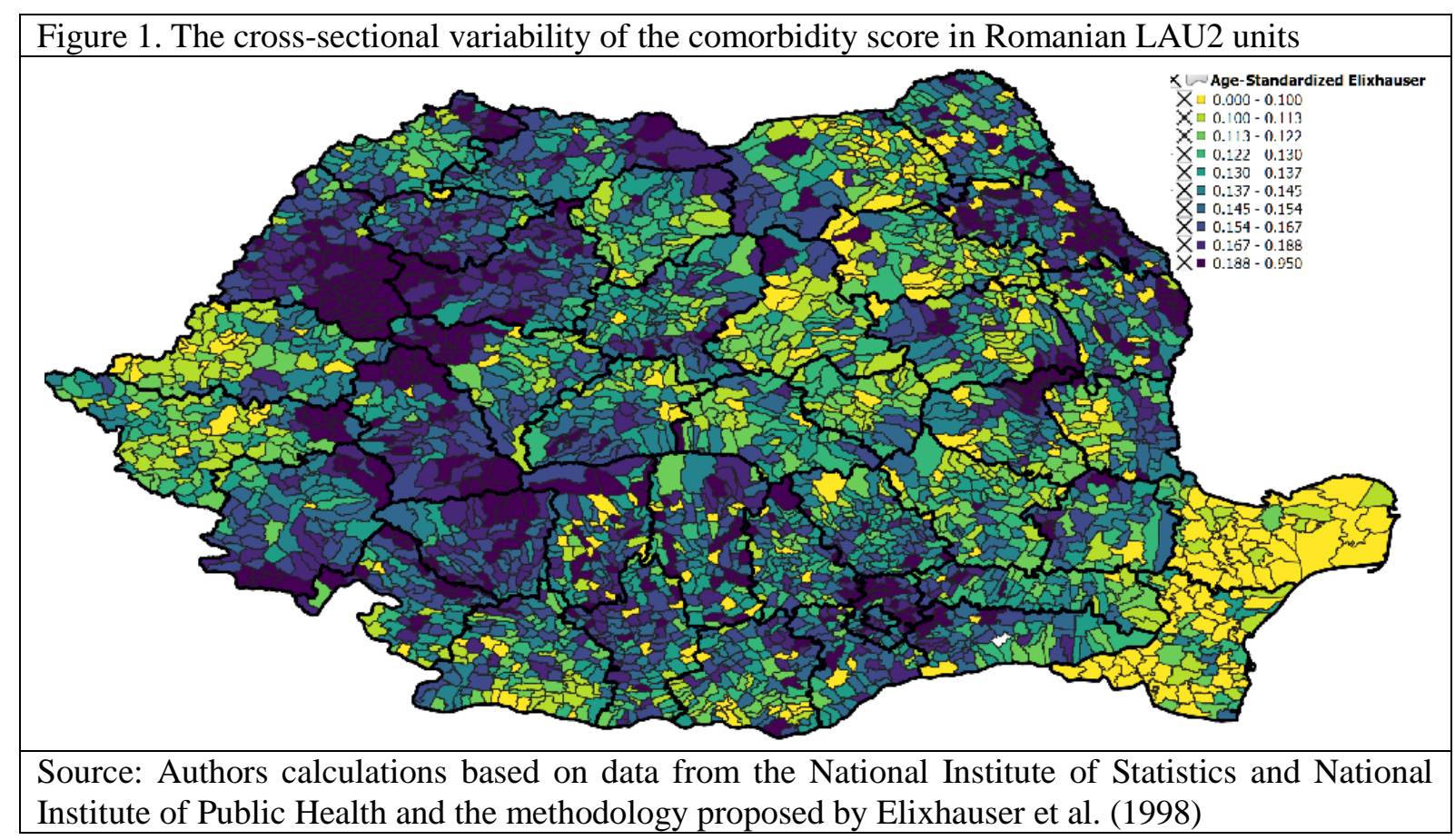

- proximity to referral hospitals as the one in the major medical university centers increase accessibility; this is linked with the fact that the referral system is not properly working, and the primary health care sector is not acting as a real gate-keeper;

- increased availability of health-related diagnostic technology amplify the chances to diagnose more diseases even in very early stages;

- several disease coding patterns (up-coding and under-coding practices) are found in hospital settings mainly linked with hospital prospective payments and this impacts directly on comorbidity accounting;

For the farthest LAU2, located at more than $90 \mathrm{~km}$ from a county residential center, it seems that in the non-MUC counties, we have a higher average of EI. This may be related to multiple local factors such as isolation, and very low availability of primary health care services. These things may contribute to an increase in multimorbidity.

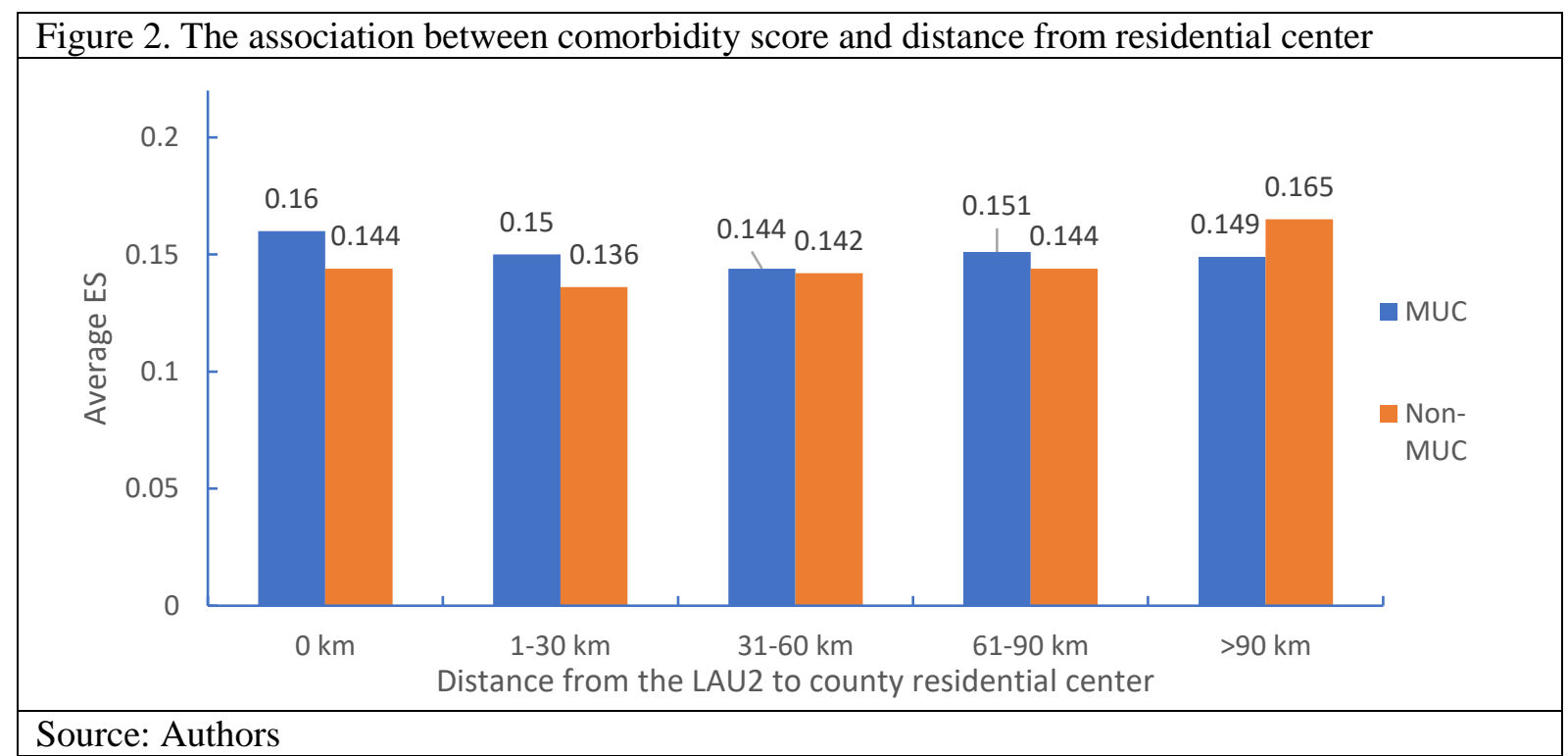

In terms of heterogeneity, the coefficient of variation is very close in both groups analyzed here MUC vs. Non-MUC, but in the MUC the variation is lower since $\mathrm{CV}=0.30$ while in the Non-MUC has a 0.33 value. 


\section{Conclusion}

There is a high variability of comorbidities at the local level (LAU2) although the difference between the ninth and first decile is below 2. There are areas that concentrate a high number of comorbidities especially around the major medical university centers and in localities from non-MUC districts located at the furthest distance from the county residential center. Proximity to referral hospitals, increased accessibility to these hospitals due to low control at primary healthcare levels, availability of diagnostic technologies and different diagnostic coding patterns, as well as isolation and the unavailability of primary health care services are listed as possible factors that can explain the results.

\section{References}

Alonso-Morán, E., Nuño-Solinis, R., Onder, G., \& Tonnara, G. (2015) Multimorbidity in risk stratification tools to predict negative outcomes in adult population, European Journal of Internal Medicine, 26, 182-189. https://doi.org/10.1016/j.ejim.2015.02.010

Brennan, P., Perola, M., van Ommen, G-J., \& Riboli, E. (2017) Chronic disease research in Europe and the need for integrated population cohorts. European Journal of Epidemiology, 32, 741-749, https://doi.org/10.1007/s10654-017-0315-2

Charlson, M., Sztrowsky, T.P., Peterson, J., \& Gold, J. (1994) Validation of a combined comorbidity index. Journal of Clinical Epidemiology, 47(11), 1245-1251. https://doi.org/10.1016/j.jphys.2016.05.008

Dicker, D., Nguyen, G., Abate, D., Abate, K.H., Abbafati, C., Abbasi, N., ...Murray, C.J.L. (2018) Global, regional, and national age-sex-specific mortality and life expectancy, 1950-2017: A systematic analysis for the Global Burden of Disease Study 2017. The Lancet, 392, 1684-1735, https://doi.org/10.1016/S0140-6736(18)31891-9

EHIG (2019) European Helath Information Gateway, https://gateway.euro.who.int/en/hfa-explorer/ accessed on 14.03.2019

Elixhauser, A., Steiner, C, Harris, D.R, \& Coffey, R.M. (1998) Comorbidity measures for use with administrative data. Medical Care, 36(1), 8-27, https://journals.lww.com/lwwmedicalcare/Abstract/1998/01000/Comorbidity_Measures_for_Use_with_Administrative.4.aspx

IHME (2019) Institute of Health Metrics and Evaluation, Global Health Data Exchange, Romania, http://www.healthdata.org/romania accessed on 14.03.2019

NIS (2019) National Institute of Statistics, Romania $\mathrm{http} / / / \mathrm{www}$. insse.ro/cms/sites/default/files/com_presa/com_pdf/sanatate2015e_0.pdf accessed on 14.03.2019

Kyu, H. H., Abate, D., Abate, K. H., Abay, S.M, Abbafati, C., Abbasi, N., ...Murray, C.J.L. (2018) Global, regional, and national disability-adjusted life-years (DALYs) for 359 diseases and injuries and healthy life expectancy (HALE) for 195 countries and territories, 1990-2017: a systematic analysis for the Global Burden of Disease Study 2017. The Lancet, 392, 1859-922, https://doi.org/10.1016/S0140-6736(18)32335-3

Ou, HT., Mukherjee, B., Erickson, S. R., Piette, J. D., Bagozzi, R. P., \& Balkrishnan, R. (2012). Comparative performance of comorbidity indices in predicting health care-related behaviors and outcomes among Medicaid enrollees with type 2 diabetes. Population health management, 15(4), 220-229. https://doi.org/10.1089/pop.2011.0037

Putnam, K.G., Buist, D.S., Fishman, P., Andrade, S.E., Boles, M., Chase, G.A., ..., Arnold Chan, K. (2002) Chronic disease score as a predictor of hospitalization. Epidemiology, 13(3), 340-346,

https://journals.lww.com/epidem/Fulltext/2002/05000/Chronic_Disease_Score_as_a_Predictor_of.16.aspx 